\title{
Managing recurrent ovarian cancer in daily clinical practice: case studies and evidence review with a focus on the use of trabectedin
}

\author{
Domenica Lorusso1, Antonio González-Martín² \& Isabelle Ray-Coquard*,3 \\ ${ }^{1}$ Fondazione Policlinico Universitario A Gemelli IRCCS, Rome, Italy \\ ${ }^{2}$ Clínica Universidad de Navarra, Madrid, Spain \\ ${ }^{3}$ Centre Leon Bérard, Laboratoire HESPER Université Claude Bernard Lyon 1, Lyon, France \\ *Author for correspondence: isabelle.ray-coquard@|yon.unicancer.fr
}

Following the failure of first-line platinum-based chemotherapy in ovarian cancer, options for further therapy in potentially platinum-responsive patients are: carboplatin doublets with pegylated liposomal doxorubicin, gemcitabine or paclitaxel in association with bevacizumab, followed by maintenance with bevacizumab (for nonpretreated patients); or maintenance monotherapy with a poly(ADP-ribose) polymerase inhibitor after a response. The choice of biological therapy depends on a patient's previous treatments and priority for a symptomatic response. In cases of a rapidly growing tumor or need for symptomatic relief, the addition of bevacizumab should be considered. Patients with limited potential sensitivity to platinum, such as those with a platinum treatment-free interval of 6-12 months, may benefit from intercalation with trabectedin and pegylated liposomal doxorubicin to possibly restore platinum sensitivity.

First draft submitted: 5 November 2020; Accepted for publication: 7 December 2020; Published online: 23 December 2020

Keywords: nonplatinum chemotherapy • ovarian cancer • platinum rechallenge $\bullet$ recurrent $\bullet$ trabectedin

In this article, three case studies and supporting evidence are presented to illustrate how various clinical factors influence the treatment approach in patients with recurrent ovarian cancer, with a focus on the use of trabectedin in situations where a nonplatinum regimen is indicated.

\section{Case study 1: when platinum rechallenge might be justified}

Case 1 involves a 43-year-old woman with a family history of hereditary breast-ovarian cancer syndrome who presented after developing bowel symptoms. A CT scan (with contrast medium) of the chest and abdomen performed in October 2013 revealed an ascitic effusion with a pelvic mass measuring approximately $14 \times 10 \mathrm{~cm}$, diffuse abdominal lymphadenopathy and omental cake with involvement of the glissonian sheath and gastric fundus. After mass biopsy, the diagnosis was FIGO stage IIIC high-grade serous ovarian cancer.

The gynecological interdisciplinary care group deemed the patient unsuitable for primary cytoreductive surgery. Consensus treatment was neoadjuvant chemotherapy, with assessment after three cycles for possible interval debulking surgery (IDS) if the response was good. After 3 cycles of neoadjuvant carboplatin and paclitaxel chemotherapy, the patient had a partial biochemical response (CA-125 levels decreased from $9100 \mathrm{IU} / \mathrm{ml}$ to $2300 \mathrm{U} / \mathrm{ml}$ ) and a partial radiological response (reduction in ascitic effusion and pelvic mass size to $6 \times 6 \mathrm{~cm}$ ). IDS was performed in January 2014, but with residual disease in the form of peritoneal nodules $(<1 \mathrm{~cm})$ on the small bowel. During surgery, the patient received hyperthermic intraperitoneal chemotherapy consisting of cisplatin $177 \mathrm{mg}$ and paclitaxel $310 \mathrm{mg}$ at $42.5^{\circ} \mathrm{C}$ for 90 min within the context of a clinical trial.

In March-April 2014 she received 3 cycles of carboplatin and paclitaxel chemotherapy. Her family history indicated the need for genetic counselling. Genetic testing identified a heterozygous BRCA1 mutation. Twelve months after completing chemotherapy (platinum-free interval [PFI] 12 months) the patient experienced disease progression in the form of multiple enlarged lymph nodes including mediastinal and porta hepatis nodes. She was considered unsuitable for secondary surgery. 
Evidence review: options for platinum-based therapy

The first step in selecting treatment for patients with recurrent ovarian cancer is to determine whether platinumbased therapy is an option. In patients potentially sensitive to platinum based on a PFI $>6$ months, options for further platinum-based therapy are carboplatin doublets with paclitaxel, gemcitabine or pegylated liposomal doxorubicin (PLD). Available evidence at the time (April 2015) indicated that adding bevacizumab to platinumbased chemotherapy significantly increased progression-free survival (PFS) in platinum-sensitive patients with no exposure to bevacizumab in the first line $[1,2]$. PARP inhibitors (PARPi) were not yet available for use.

\section{Case continuation}

The patient was treated in the second line with 6 cycles of carboplatin (area under curve 4; days 1 and 21) and gemcitabine $\left(1000 \mathrm{mg} / \mathrm{m}^{2}\right)$ plus bevacizumab $(15 \mathrm{mg} / \mathrm{kg})$. At treatment end (November 2015) a radiological complete response was observed and bevacizumab maintenance therapy $(15 \mathrm{mg} / \mathrm{kg})$ commenced. In January 2017, her CA-125 level increased. A CT scan revealed disease progression with enlarged lymph nodes, peritoneal carcinomatosis and ascites. PFI was 14 months. The patient was again considered unsuitable for secondary surgery.

\section{Evidence review: olaparib maintenance therapy in patients with $B R C A$ mutations}

At the time of disease progression in 2017, a Phase II trial of olaparib maintenance therapy in patients with $B R C A$-mutated platinum-sensitive recurrent ovarian cancer who were in response to platinum reported significant improvement in PFS and overall survival (OS) compared with placebo $[3,4]$. Predictive factors for prolonged PFS to olaparib maintenance therapy in patients with $B R C A$-mutated ovarian cancer include a complete or partial response to previous platinum-based therapy and a PFI $>12$ months [5].

\section{Case continuation}

Key factors informing the selection of third-line treatment in our patient were a BRCA mutation, prolonged PFI (>12 months) after second-line therapy and high probability of responding to platinum rechallenge. Our selection was carboplatin and paclitaxel followed by olaparib in the event of a response. (Note: had evidence about the efficacy of PLD retreatment been available at the time of third recurrence, as is currently available, carboplatin and PLD would have been chosen instead because of its superior toxicity profile). In May 2017, after 4 of 6 planned cycles of carboplatin and paclitaxel, chemotherapy was discontinued due to adverse effects: grade 2 peripheral neuropathy and grade 4 febrile neutropenia. Based on a partial response at all disease sites and decreased CA-125 levels, olaparib maintenance therapy (400 mg twice daily) commenced.

Evidence review: duration of maintenance therapy for platinum-sensitive recurrent ovarian cancer A real-world retrospective study of 3629 patients with platinum-sensitive ovarian cancer reported that time to next treatment was significantly $(\mathrm{p}<0.0125)$ prolonged with PARPi $(\mathrm{n}=151)$ versus bevacizumab $(\mathrm{n}=212)$ maintenance therapy [6]. Elsewhere, a network meta-analysis of patients with platinum-sensitive recurrent ovarian cancer treated with bevacizumab $(\mathrm{n}=1563)$ or PARPi $(\mathrm{n}=1839)$ found that PARPi improved PFS compared with bevacizumab based on indirect comparison (hazard ratio [HR] $=0.70,95 \%$ CI: 0.54-0.91) [7].

Although both studies suggested that outcomes are better with PARPi than bevacizumab maintenance therapy in platinum-sensitive recurrent ovarian cancer, such conclusions cannot be drawn due to important differences in their use. To qualify for maintenance therapy with PARPi, patients must be in response to platinum. Conversely, bevacizumab is combined with chemotherapy from the start of treatment for recurrent disease without patient preselection according to platinum response. This concept is better understood by comparing the designs of bevacizumab and PARPi maintenance studies in platinum-sensitive patients. In the OCEANS trial of bevacizumab, patients who relapsed after first-line platinum-based treatment were immediately randomized to receive platinumbased chemotherapy plus either bevacizumab or placebo [1]. In the olaparib study, patients were randomized to receive olaparib or placebo at the end of chemotherapy only if they had achieved a response to their platinum-based regimen [3]. These methodological differences imply different and better prognoses among PARPi-treated patients and preclude making comparisons.

\section{Case continuation}

In December 2017, a gradual increase was detected in the patient's CA-125 level during olaparib maintenance therapy. A CT scan showed lymph node progression. PFI was 7 months. 
17/02/2018: restaging CT scan after three cycles

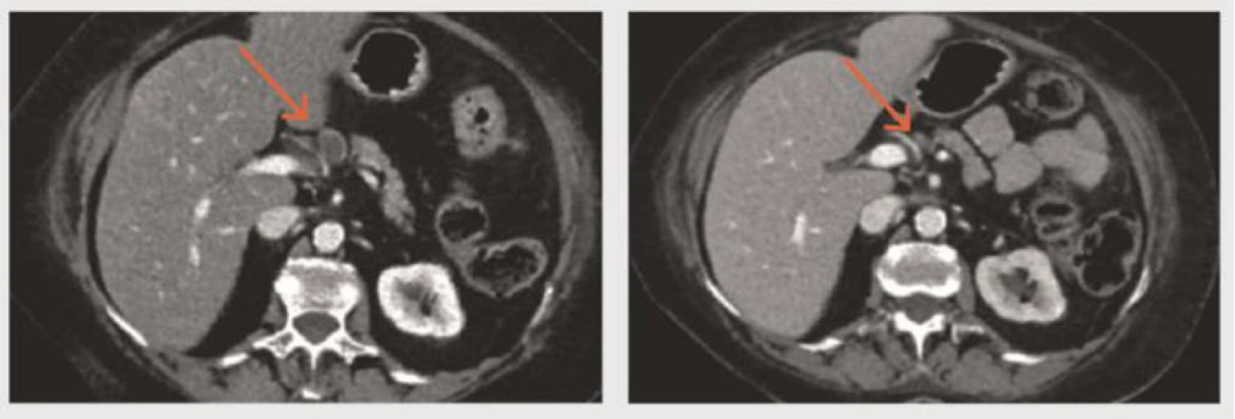

Figure 1. Restaging computed tomography scan after three cycles of trabectedin and pegylated liposomal doxorubicin in the fourth line in a patient with recurrent ovarian cancer.

CT: Computed tomography.

\section{Evidence review: later line treatment options}

Based on a PFI of 7 months at the time of progression, carboplatin monotherapy was an option. However, the patient had already received three lines of platinum-based therapy and experienced significant toxicities in the third line. Cumulative risk of allergic reactions is known to increase with the number of platinum cycles, particularly in patients with BRCA mutations [8]. Given that ovarian cancer patients with recurrent disease achieve clinical benefit with active therapy even in later lines, best supportive care was not appropriate at this time.

In patients with platinum-sensitive recurrent ovarian cancer for whom platinum might not be the best option, an alternative is trabectedin and PLD [9]. A randomized Phase III trial showed that trabectedin and PLD was superior to PLD monotherapy in platinum-sensitive ovarian cancer patients [10,11], with no associated impairment to quality of life [12]. In BRCA-mutated patients specifically, median PFS (13.6 vs 5.5 months; $\mathrm{p}<0.0001$ ) and median OS (27.4 vs 18.7 months; $\mathrm{p}=0.0093$ ) were significantly prolonged with trabectedin and PLD compared with PLD alone [13]. An important consideration with fourth-line treatment is whether the safety profile of the proposed regimen is compatible with such an advanced line of therapy. A prospective real-world study of trabectedin and PLD in heavily pretreated patients $(\mathrm{n}=158)$ with platinum-sensitive recurrent ovarian cancer (one previous line: $25 \%$ of patients; two previous lines: $34 \%$; $\geq 3$ previous lines: $40 \%$ ) found that the safety profile of the combination was consistent with that previously observed in less pretreated patients [14].

\section{Case continuation}

A restaging CT scan in February 2018 showed a partial serological and radiological response after three cycles of trabectedin and PLD in the fourth line (Figure 1), which was maintained at the sixth and tenth cycle. Toxicities experienced by the patient were grade 2 anemia, grade 1 neutropenia, grade 2 mucositis and grade 1 transaminase elevation. In October 2018 after 10 cycles of chemotherapy, the patient requested a break from treatment. She is currently being monitored and, at the time of writing (October 2019), had shown no clinical, radiological or biochemical evidence of progression. The patient's treatment history is summarized in Figure 2.

\section{Case study 2: when symptomatic response may be a priority}

Case 2 describes a 36-year-old woman with no relevant personal or family medical history of cancer who developed a right ovarian cyst (January 2018) and abdominal distension (May 2018). CA-125 was 3623 U/ml. Ultrasound, CT and pelvic MRI performed in June 2018 showed peritoneal carcinomatosis, with retroperitoneal and enlarged pericardiophrenic lymph nodes.

A positron emission tomography (PET)-CT scan performed in July 2018 during her first consultation at a specialist cancer center revealed significant abdominal disease, with involvement of supraclavicular, mediastinal and inguinal lymph nodes. Laparoscopy, as agreed by the multidisciplinary tumor board, showed ascites $(5 \mathrm{~L})$, a left ovarian mass of $6 \mathrm{~cm}$, a frozen pelvis, omental infiltration with adhesions to the abdominal wall, infiltration of both diaphragmatic surfaces, some small bowel implants and implants on the hepatic surface, pelvic abdominal wall and presplenic area. 


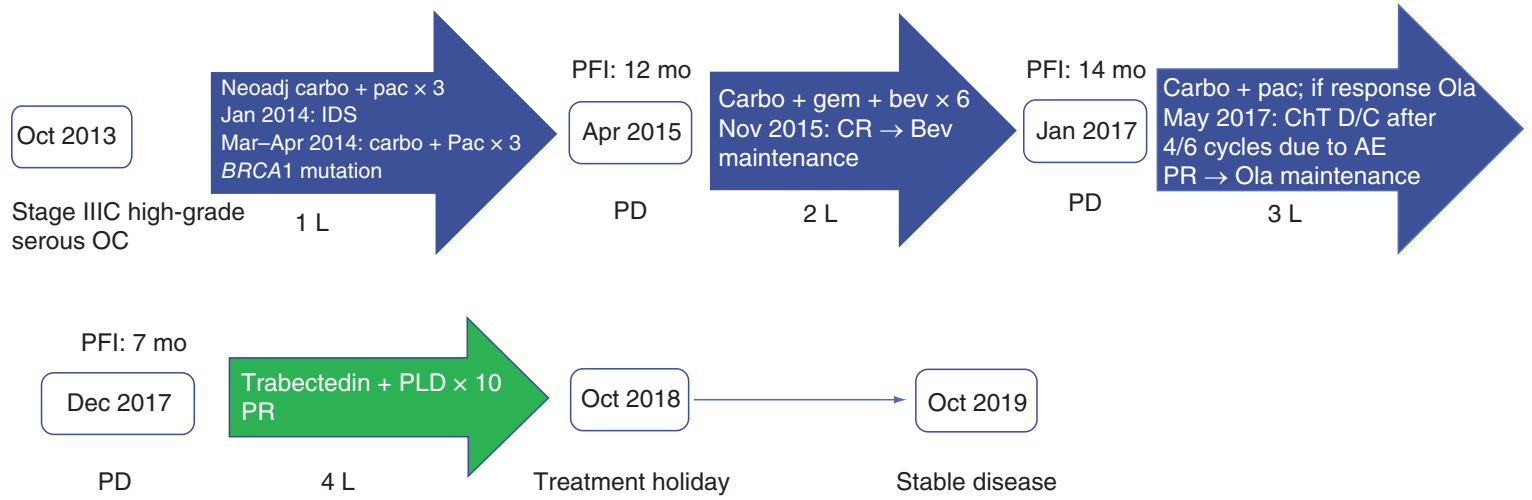

Figure 2. Case 1: schematic representation of treatment history.

1 L: First line; 2 L: Second line; 3 L: Third line; 4 L: Fourth line; AE: Adverse event; Bev: Bevacizumab; Carbo: Carboplatin; ChT: Chemotherapy; CR: Complete response; D/C: Discontinued; Gem: Gemcitabine; IDS: Interval debulking surgery; mo: Months; Neoadj: Neoadjuvant; OC: Ovarian cancer; Ola: Olaparib; Pac: Paclitaxel; PD: Progressive disease; PFI: Platinum-free interval; PLD: Pegylated liposomal doxorubicin; PR: Partial response.

Pathology identified a high-grade serous carcinoma and absent p53 immunohistochemical staining. Genetic analysis in tumor tissue of 161 cancer-associated genes (using Oncomine) identified mutations in the TP53 and BRCA2 genes. The somatic BRCA2 mutation (c. $681+1 \mathrm{G}>\mathrm{A}$ ) was observed in $4 \%$ of biopsied tumor cells. No germline mutations in $B R C A 1$ or $B R C A 2$ were identified by next-generation sequencing in peripheral blood.

Neoadjuvant carboplatin and paclitaxel ( $3 \times$ cycles) was initiated in July 2018. In September 2018, a nearcomplete response was observed. IDS performed in October 2018 resulted in optimal cytoreduction with minimal residual disease. Three additional cycles of carboplatin and paclitaxel were administered, ending in December 2018.

In early January 2019, the patient's CA-125 level was $5.8 \mathrm{U} / \mathrm{ml}$. Maintenance therapy commenced with olaparib $400 \mathrm{mg}$ twice daily.

In early March 2019, she experienced abdominal discomfort and distension. CA-125 was $912 \mathrm{U} / \mathrm{ml}$ and a CT scan showed ascites. A new molecular study in cells obtained by paracentesis showed a TP53 mutation but no $B R C A 2$ mutation, possibly due to low clonality.

\section{Evidence review: next treatment options}

According to the previous paradigm for managing recurrent ovarian cancer [15] the patient would have been categorized as platinum-resistant based on a PFI $<6$ months. However, other factors known to influence response to platinum include $B R C A$ status, tumor histology, type of follow-up, use of maintenance therapy, quality of life and global health, and performance status. RECIST (Response Evaluation Criteria In Solid Tumors) response rates to platinum-based therapy of approximately $30 \%$ have been reported in patients with a PFI $<6$ months $[16,17]$.

Updated European Society for Medical Oncology (ESMO)/European Society of Gynaecological Oncology (ESGO) consensus conference recommendations feature a therapy-oriented definition for systemic treatment of relapsed disease [9]. Circumstances in which platinum therapy might not be the best option are early symptomatic relapse, progression on prior platinum, and platinum intolerability. Circumstances in which platinum might be the best option (i.e., rechallenge is justified) are prior response and no contraindications to platinum.

In patients eligible for platinum who are potentially platinum-responsive, recommended treatments are platinumbased rechallenge followed by PARPi or platinum-based rechallenge plus bevacizumab [9]. In the absence of prior administration of either agent, the choice of one or other biological agent is based on two main factors. A PARPi is treatment of choice if there is no priority for a symptomatic response or contraindications to bevacizumab are present. Bevacizumab is treatment of choice if there is priority for a symptomatic response and contraindications to bevacizumab are absent. Two Phase III trials have shown a statistically significant and clinically meaningful improvement in PFS when bevacizumab was added to carboplatin and gemcitabine [1] and to carboplatin and paclitaxel [18], followed by bevacizumab maintenance. A recent study has shown significantly greater improvement in median PFS ( $\mathrm{HR}=0.80 ; 95 \%$ CI: 0.68-0.95) and median OS (HR = 0.81; 95\% CI: 0.66-0.98) with carboplatin and PLD plus bevacizumab compared with carboplatin and gemcitabine plus bevacizumab [19]. 


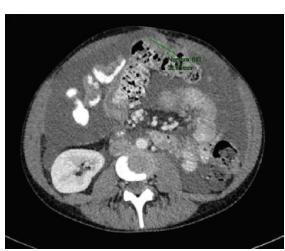

CA-125: 912 $12 / 03 / 2019$

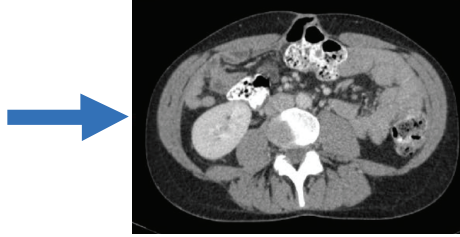

Platinum Gemcitabine Bevacizumab
CA-125: 32 $31 / 07 / 2019$

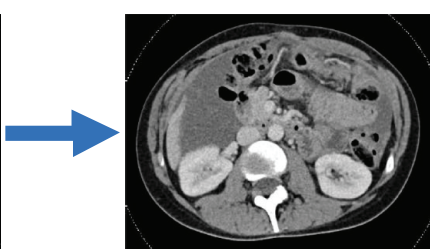

Bevacizumab maintenance
CA-125: 1311

$25 / 09 / 2019$

Figure 3. Computed tomography scans of a patient with recurrent ovarian cancer and previous exposure to olaparib during rechallenge with platinum and gemcitabine plus bevacizumab.

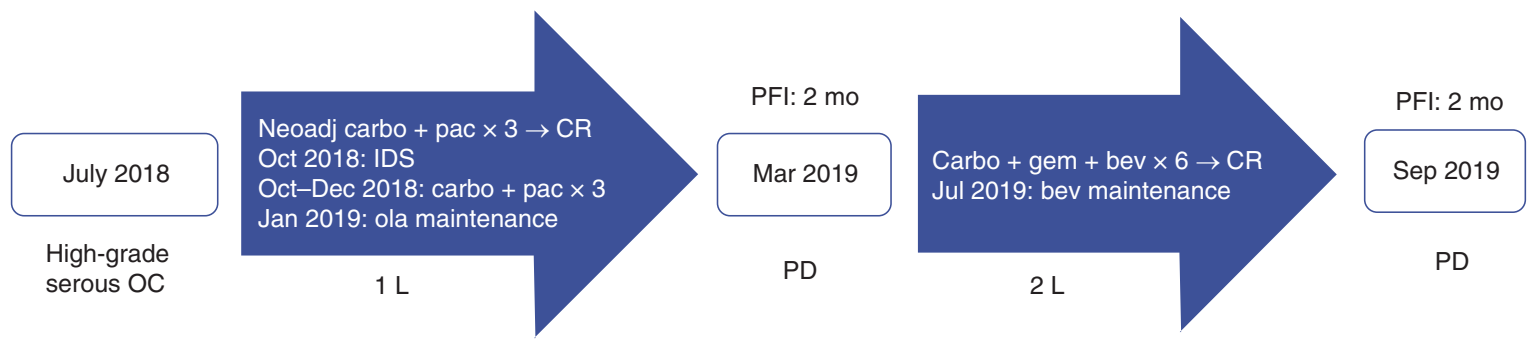

Figure 4. Case 2: schematic representation of treatment history.

1 L: First line; 2 L: Second line; Bev: Bevacizumab; Carbo: Carboplatin; CR: Complete response; Gem: Gemcitabine; IDS: Interval debulking surgery; mo: Months; Neoadj: Neoadjuvant; OC: Ovarian cancer; Ola: Olaparib; Pac: Paclitaxel; PD: Progressive disease; PFI: Platinum-free interval.

\section{Case continuation}

As the patient was young, symptomatic and had achieved a complete response after 3 cycles of platinum-based treatment, she was a candidate for platinum rechallenge. In view of her previous exposure to a PARPi, she received platinum and gemcitabine plus bevacizumab. Treatment began in March 2019. After 6 cycles (July 2019) she had a complete response, with symptom resolution and no evidence of disease by CT (Figure 3). CA-125 was $32 \mathrm{U} / \mathrm{ml}$. Bevacizumab maintenance therapy commenced but, two months later (by September 2019), she progressed with peritoneal carcinomatosis. CA-125 was $1311 \mathrm{U} / \mathrm{ml}$. The patient's treatment history is summarized in Figure 4.

\section{Case study 3: managing an early symptomatic relapse}

A 63-year-old patient presented in July 2012 (outside of Centre Leon Bérard) with abdominal pain and iliac masses. A CT scan showed a mesenteric mass $(13.9 \mathrm{~cm})$, heterogeneous uterus and hepatic lesions. She had a family history (mother, sister) of ovarian cancer. Her CA-125 was $9922 \mathrm{U} / \mathrm{ml}$.

A laparoscopy performed in August 2012 revealed peritoneal carcinomatosis on the diaphragm (right and left sides), Glisson's capsule and pelvic masses. Multiple biopsies confirmed a high-grade serous carcinoma. Immunochemistry indicated a TP53-mutated $\left(\mathrm{p} 53^{+}\right)$Wilms Tumor (WT1+), positive for estrogen and progesterone receptors, favoring an ovarian localization.

\section{Evidence review: treatment options for non-optimally resectable ovarian cancer}

An initial consideration for all patients with a new diagnosis of ovarian cancer is the possibility of surgery. According to ESMO/ESGO consensus recommendations [9], conditions ruling out primary surgery include:

- Diffuse deep infiltration of the root of small bowel mesentery;

- Diffuse carcinomatosis of the small bowel with involvement of large parts that would require resection leading to short bowel syndrome (remaining bowel $<1.5 \mathrm{~m}$ );

- Diffuse involvement/deep infiltration of the stomach/duodenum and/or head or middle part of the pancreas;

- Involvement of coeliac trunk, hepatic arteries, left gastric artery;

- Central or multisegmental parenchymal liver metastases; 


$\begin{aligned} & \text { Table 1. Factors informing the decision to offer platinum-based therapy or nonplatinum treatment to a patient with } \\
& \text { relapsed ovarian cancer (case 3). }\end{aligned}$
\begin{tabular}{ll} 
Factors to consider & Patient's characteristics \\
\hline Tumor biology & Unknown BRCA status (as at April 2014) \\
\hline Histology & Serous carcinoma \\
\hline Prior therapies & Carboplatin/paclitaxel/bevacizumab, IDS and treatment re-introduction \\
\hline TFlp & $<1$ year \\
\hline Prior response to chemotherapy & Suboptimal response to neoadjuvant chemotherapy plus bevacizumab and IDS \\
\hline Persistent toxicity & Grade 2 neuropathy \\
\hline Patient preference or potential compliance with treatment & Previous refusal of maintenance therapy \\
\hline $\begin{array}{l}\text { IDS: Interval debulking surgery; TFlp: Treatment-free interval since last platinum. } \\
\text { Data taken from [9]. }\end{array}$ & \\
\hline
\end{tabular}

- Multiple parenchymal lung metastases (preferable histologically proven);

- Nonresectable lymph nodes;

- Brain metastases.

In patients with nonoptimally resectable ovarian cancer, neoadjuvant carboplatin and paclitaxel is standard treatment before IDS. Use of bevacizumab in neoadjuvant chemotherapy is open to discussion in the multidisciplinary tumor board [9]. Two randomized Phase II studies support the addition of bevacizumab (3-4 cycles) to neoadjuvant chemotherapy (four cycles of carboplatin and paclitaxel) in initially unresectable ovarian cancer [20,21]. The ANTHALYA trial reported a significantly higher rate of complete resection with added bevacizumab from 4 to 5 weeks before and for 7 weeks after, IDS compared with the reference rate (58.6 vs 45\%) [20]. In the GEICO 1205 trial, adding bevacizumab in the neoadjuvant setting ( 6 weeks before and 6 weeks after IDS) improved surgical operability without increasing toxicity. The proportion of patients undergoing surgery in the chemotherapy arm versus chemotherapy plus bevacizumab arm was 67 and 89\%, respectively $(p=0.029)$ [21].

\section{Case continuation}

In accordance with ESMO/ESGO recommendations, initial debulking surgery was not performed. Neoadjuvant chemotherapy with carboplatin and paclitaxel plus bevacizumab was administered. The patient experienced grade 2 asthenia, alopecia, infectious pneumonia and grade 2 neuropathy which persisted until progression. In February 2013, after 6 cycles of neoadjuvant treatment, radical debulking surgery was performed consisting of hysterectomy, bilateral anexectomies, and infragastric omentectomy. Biopsies were positive for the ileum, mesenteric, colon and pouch of Douglas, but negative for the diaphragm and paracolic gutters. Although the surgeon considered the resection to be a complete cytoreduction (CC0), histological review indicated minimal residual disease.

Chemotherapy plus bevacizumab $(2 \times$ cycles $)$ was reintroduced in March 2013. Regular evaluation indicated a normal CA-125 level and normal CT scan, prompting the patient to request treatment discontinuation. Maintenance therapy was refused.

In April 2014, 10 months after treatment end, the patient relapsed. CA-125 was $485 \mathrm{U} / \mathrm{ml}$. A CT scan showed at least two tumor masses which were confirmed by PET-CT.

\section{Evidence review: next treatment options}

Factors to consider when deciding whether to offer platinum-based therapy or nonplatinum treatment to a patient with relapse are tumor biology, tumor histology, prior therapies, treatment-free interval since last platinum (TFIp), prior response to chemotherapy, persistent toxicities, and patient preference [9]. Time elapsed since last platinum continues to have prognostic value since it represents a continuum of probability of response to further chemotherapy, although not all patients who achieve a TFIp $>6$ months respond to platinum rechallenge. Objective response rates ranged from 47.2 to $66 \%$ in patients with a TFIp $>6$ months, and from 27 to $33 \%$ in patients with a TFIp of 6 to 12 months [9,22]. Taking all factors into consideration (Table 1), it was concluded that platinum-based treatment might not be the best option for our patient.

The nonplatinum combination of trabectedin and PLD has shown superior efficacy to PLD monotherapy in ovarian cancer patients who relapse beyond 6 months to platinum-based treatment (Table 2) [10], with no detriment to quality of life [12]. Trabectedin and PLD has shown consistent efficacy in patients with questionable 
Table 2. Efficacy results from the randomized Phase III study OVA-301 in patients with platinum-sensitive ovarian cancer (relapse $\geq 6$ months after first-line platinum-based therapy).

\begin{tabular}{|c|c|c|c|}
\hline Efficacy end points & PLD monotherapy & Trabectedin + PLD & p-value \\
\hline Median PFS & 7.5 months & 9.2 months & 0.0170 \\
\hline Median OS ${ }^{\dagger}$ & 24.1 months & 28.4 months & 0.0319 \\
\hline Response rate & $22.6 \%$ & $35.3 \%$ & 0.0042 \\
\hline
\end{tabular}

$\dagger$ Based on Cox regression analysis after adjustment for key prognostic factors.

OS: Overall survival; PFS: Progression-free survival; PLD: Pegylated liposomal doxorubicin.

Data taken from $[10,11]$.

\begin{tabular}{|c|c|c|c|c|}
\hline \multicolumn{2}{|c|}{ Early symptomatic relapse ( 6 to 12 months) to previous platinum } & \multicolumn{2}{|c|}{ No complete response to previous platinum } & \multirow{2}{*}{$\begin{array}{r}\text { Ref. } \\
{[10,23]}\end{array}$} \\
\hline $\begin{array}{l}49.6 \% \text { of platinum-sensitive patients } \\
\text { in OVA-301 }(n=214)\end{array}$ & $\begin{array}{l}\text { Median PFS: } \\
\text { - Trabectedin/PLD: } 7.4 \text { months } \\
\text { - PLD: } 5.5 \text { months; } p=0.0152\end{array}$ & $\begin{array}{l}41.4 \% \text { of platinum-sensitive patients } \\
\text { in OVA-301 }(n=178)\end{array}$ & $\begin{array}{l}\text { Median PFS: } \\
\text { - Trabectedin/PLD: } 9.6 \text { months } \\
\text { - PLD: } 5.5 \text { months; } p=0.0089\end{array}$ & \\
\hline & $\begin{array}{l}\text { Median OS: } \\
\text { - Trabectedin/PLD: } 22.4 \text { months } \\
\text { - PLD: } 16.4 \text { months; } \mathrm{p}=0.0027\end{array}$ & & $\begin{array}{l}\text { Median OS: } \\
\text { - Trabectedin/PLD: } 25.0 \text { months } \\
\text { - PLD: } 19.4 \text { months; } p=0.0315\end{array}$ & [24] \\
\hline
\end{tabular}

OS: Overall survival; PFS: Progression-free survival; PLD: Pegylated liposomal doxorubicin.

Data taken from $[10,23,24,25]$.

platinum sensitivity regardless of the manner in which sensitivity was measured: early symptomatic relapse (612 months) [23,24] or no complete response to previous platinum-based therapy (Table 3) [25]. The combination of trabectedin and PLD is well-tolerated, often allowing patients to resume normal daily activities [26]. Trabectedin and PLD is not associated with cumulative end organ toxicities [27] or with disturbing side effects such as alopecia, hypersensitivity reactions, hand-foot syndrome or mucositis [25] and is compatible with long-term exposure, having been shown to provide clinical benefit for up to 21 cycles [28]. Importantly, use of trabectedin and PLD allows patients time to recover from the impact of previous platinum-based therapy [29], opening up the possibility of future platinum rechallenge.

\section{Case continuation}

From May to July 2014, the patient received trabectedin and PLD ( $4 \times$ cycles) in the second line and achieved a partial response. She experienced grade 2 nausea, grade 3 neutropenia, and grade 2 asthenia during the first cycle; grade 1 nausea and grade 1 asthenia during the second cycle (managed with corticosteroids $\times 5$ days and G-CSF support); and interstitial pneumopathy during cycle 4 (asthmatic patient). She requested a treatment break during the holidays. Genetic screening indicated no germline BRCA mutation.

A CT scan in November 2014 detected an increase in the size of a residual carcinomatosis lesion to $16 \mathrm{~mm}$. Trabectedin and PLD was restarted. In February 2015, after 4 cycles of chemotherapy, a complete response was observed. The patient was asymptomatic, and a CT scan revealed no residual disease. Treatment was discontinued due to a pulmonary infectious event, although bacterial test results were negative. A CT scan in August 2015 showed ascites without identification of target lesions and subocclusion syndrome.

\section{Evidence review: next treatment options}

The trabectedin and PLD combination has shown increased survival when platinum is the subsequent treatment. In patients from the OVA-301 study with limited platinum sensitivity (PFI of 6-12 months; $\mathrm{n}=214$ ), median OS was 22.4 months with trabectedin and PLD and 16.4 months with PLD monotherapy (HR = 0.64; $\mathrm{p}=0.0027)$ [23]. The differential in median OS was greater among patients $(\mathrm{n}=94)$ who received platinum-based chemotherapy as the next treatment after trabectedin + PLD or PLD (27.7 vs 18.7 months; HR 0.58; $\mathrm{p}=0.0153$ ) [24], possibly because trabectedin restores platinum sensitivity in ovarian cancer by selecting nucleotide excision repair-deficient cells from the heterogeneous tumor cell population that are more responsive to platinum [30]. ESMO/ESGO consensus recommendations state that, following treatment with a nonplatinum regimen (in combination or as monotherapy), platinum rechallenge can be considered if the patient had not progressed during prior platinum therapy [9]. 


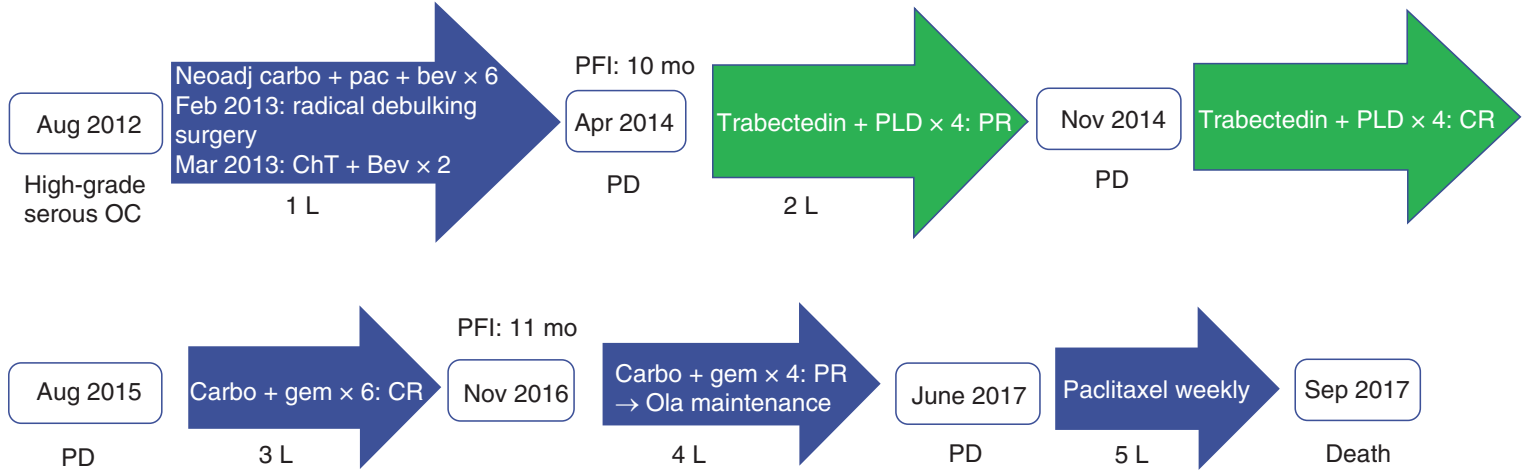

Figure 5. Case 3: schematic illustration of treatment history.

1 L: First line; 2 L: Second line; 3 L: Third line; 4 L: Fourth line; 5 L: Fifth line; Bev: Bevacizumab; Carbo: Carboplatin; ChT: Chemotherapy; CR: Complete response; Gem: Gemcitabine; mo: Months; Neoadj: Neoadjuvant; OC: Ovarian cancer; Ola: Olaparib; Pac: Paclitaxel; PD: Progressive disease; PFI: Platinum-free interval; PLD: Pegylated liposomal doxorubicin; PR: Partial response.

\section{Case continuation}

The patient received carboplatin plus gemcitabine $(6 \times$ cycles $)$ in the third line and achieved a complete response. Toxicities were grade 4 anemia requiring two red blood cell transfusions; grade 4 neutropenia associated with Escherichia coli infection which resolved with antibacterial therapy; allergic reaction to carboplatin after cycle 2 (premedication was used subsequently without allergic reaction); grade 2 liver toxicity and grade 2 neuropathy.

In November 2016, 11 months after the end of platinum-based rechallenge, a CT scan showed multiple carcinomatosis lesions and ascites. Subocclusion syndrome resolved completely after 2 days' treatment with corticosteroids.

Genetic tumor screening identified a somatic BRCA1 mutation. Four cycles of carboplatin and gemcitabine were administered in the fourth line and a partial response was observed. Olaparib maintenance treatment was initiated, which was associated with grade 3 diarrhea, grade 2 headache, grade 2 nausea and grade 1 asthenia. Despite a reduction in the olaparib dose, the patient continued to report adverse events and discontinued the PARPi after 4 months. Two months later, clinical and radiological progression were observed. Weekly paclitaxel treatment commenced, but the patient died after 3 months of chemotherapy due to disease progression. The patient's treatment history is summarized in Figure 5.

\section{Conclusion}

The case studies illustrate how disease- and patient-related factors can inform treatment selection following the failure of first-line platinum-based chemotherapy in patients with recurrent ovarian cancer.

Case 1 describes a young woman with advanced stage disease at diagnosis. A PFI of 14 months after second-line platinum-based chemotherapy indicated a high probability of response to platinum rechallenge, while previous exposure to bevacizumab and a BRCA mutation informed the decision to use PARPi maintenance therapy in the event of a response. A PFI of 7 months after third-line platinum-based treatment and olaparib suggested that carboplatin monotherapy might be an option for fourth-line treatment; however, the significant toxicity the patient experienced during third-line treatment ruled in favor of a nonplatinum combination. The patient received ten cycles of trabectedin and PLD before requesting a break from treatment. One year later she continued to show no evidence of progression.

Case 2 involves a young woman with $B R C A$-mutated disease. Despite a PFI of only 2 months at the time of relapse to first-line platinum-based chemotherapy, other factors such as $B R C A$ status, a near complete response and symptomatic disease made her a candidate for platinum rechallenge along with bevacizumab maintenance due to first-line exposure to olaparib. The patient achieved a complete response and symptom resolution to second-line platinum-based chemotherapy, although she relapsed 2 months later.

The woman in case 3 had a PFI of 10 months at the time to relapse to first-line platinum-based chemotherapy. The questionable sensitivity to platinum together with other disease- and patient-related factors pointed to a nonplatinum regimen as a better option. Trabectedin and PLD (10× cycles) led to 15 months of disease control and no residual disease. She subsequently achieved a complete response to third-line platinum-based chemotherapy 
with a PFI of 11 months until next relapse, possibly reflecting restored platinum sensitivity after intercalation with trabectedin and PLD.

\section{Executive summary}

- Three case studies illustrate how disease- and patient-related factors apart from the platinum-free interval (PFI) can inform treatment decisions in patients with recurrent ovarian cancer.

Case study 1: when platinum rechallenge might be justified

- In a young woman with advanced disease at diagnosis, prolonged responses to first- and second-line platinum-based chemotherapy and a BRCA mutation supported platinum rechallenge in the third line. However, early discontinuation of platinum-based therapy due to intolerable adverse effects indicated the need to switch to a nonplatinum-based regimen. Fourth-line treatment with trabectedin and pegylated liposomal doxorubicin (PLD) was well tolerated and the patient maintained a prolonged response with no evidence of progression.

Case study 2: when symptomatic response may be a priority

- A PFI of only 2 months to first-line platinum-based chemotherapy suggested that the patient was platinum resistant. However, the combined weight of other factors such as young age, a complete response to first-line treatment and symptomatic relapse pointed to platinum rechallenge as the most appropriate approach. The patient achieved a complete response and symptom resolution to second-line platinum-based chemotherapy, although relapsed 2 months later.

Case study 3: managing an early symptomatic relapse

- Although platinum rechallenge to manage relapse was also an option for case 3 with a PFI of 10 months after first-line platinum-based chemotherapy, additional factors informed the decision to use a nonplatinum regimen. The patient achieved a complete response (15 months of disease control with no residual disease) to trabectedin and PLD. Third-line platinum-based chemotherapy led to a prolonged complete response, possibly reflecting restored platinum sensitivity after intercalation with trabectedin and PLD.

\section{Author contributions}

Collection and/or assembly of data was done by D Lorusso, A González-Martín, I Ray-Coquard. Manuscript writing was performed by D Lorusso, A González-Martín, I Ray-Coquard. Final approval of manuscript was also done by D Lorusso, A González-Martín, I Ray-Coquard.

Financial \& competing interests disclosure

D Lorusso has acted as an advisory board member for Amgen, AstraZeneca, Clovis, Genmab, Immunogen, Merck, PharmaMar, Roche and Tesaro; has received institutional grants for research from Clovis, Merck, PharmaMar, Roche and Tesaro; has provided expert testimony for Clovis; is a principal investigator of registered trials for AstraZeneca, Clovis, Genmab, Immunogen. Merck, PharmaMar, Roche and Tesaro; has received travel support from AstraZeneca, PharmaMar, Roche and Tesaro and has nonfinancial interests as a member of the European Network for Gynaecological Oncological Trial groups (ENGOT), European Society of Gynaecological Oncology (ESGO), European Society for Medical Oncology (ESMO), Gynecologic Cancer Intergroup (GCIG; Board of Directors), Italian Association of Medical Oncology (AIOM) and Multicenter Italian Trials in Ovarian Cancer (MITO). A González-Martín has received grants for lectures or advisory roles from AstraZeneca, Clovis, Genmab, GSK, ImmunoGen, Merck, MSD, Novartis, Novocure, PharmaMar, Pfizer, Roche and Tesaro. I Ray-Coquard has received honoraria from AstraZeneca, Clovis, PharmaMar and Tesaro; has received consulting/advisory board fees from AstraZeneca, Clovis, Genmab, MSD, PharmaMar, Pfizer, Roche and Tesaro; has received research funding from MSD; has received travel expenses from AstraZeneca, PharmaMar, Roche and Tesaro. The authors have no other relevant affiliations or financial involvement with any organization or entity with a financial interest in or financial conflict with the subject matter or materials discussed in the manuscript apart from those disclosed.

Editorial assistance was provided by Robert Furlong and Kerry Dechant on behalf of Content Ed Net (Madrid, Spain) with funding from PharmaMar (Madrid, Spain).

Ethical conduct of research

The authors state that they have obtained verbal and written informed consent from the patient/patients for the inclusion of their medical and treatment history within this case report.

Open access

This work is licensed under the Attribution-NonCommercial-NoDerivatives 4.0 Unported License. To view a copy of this license, visit http://creativecommons.org/licenses/by-nc-nd/4.0/ 


\section{References}

Papers of special note have been highlighted as: $\bullet$ of interest; $\bullet \bullet$ of considerable interest

1. Aghajanian C, Blank SV, Goff BA et al. OCEANS: a randomized, double-blind, placebo-controlled Phase III trial of chemotherapy with or without bevacizumab in patients with platinum-sensitive recurrent epithelial ovarian, primary peritoneal, or fallopian tube cancer. $J$. Clin. Oncol. 30, 2039-2045 (2012).

2. Aghajanian C, Goff B, Nycum LR, Wang YV, Husain A, Blank SV. Final overall survival and safety analysis of OCEANS, a Phase III trial of chemotherapy with or without bevacizumab in patients with platinum-sensitive recurrent ovarian cancer. Gynecol. Oncol. 139, 10-16 (2015).

3. Ledermann J, Harter P, Gourley C et al. Olaparib maintenance therapy in patients with platinum-sensitive relapsed serous ovarian cancer: a preplanned retrospective analysis of outcomes by BRCA status in a randomised Phase II trial. Lancet Oncol. 15, 852-861 (2014).

4. Ledermann JA, Harter P, Gourley C et al. Overall survival in patients with platinum-sensitive recurrent serous ovarian cancer receiving olaparib maintenance monotherapy: an updated analysis from a randomised, placebo-controlled, double-blind, Phase II trial. Lancet Oncol. 17, 1579-1589 (2016).

5. Labidi-Galy SI, de La Motte Rouge $\mathrm{T}$ et al. Clinical factors associated with prolonged response and survival under olaparib as maintenance therapy in BRCA mutated ovarian cancers. Gynecol. Oncol. 155(2), 262-269 (2019).

- Multicenter retrospective study indicating that a platinum-free interval $\geq 12$ months, complete response and normalized CA-125 levels after ultimate platinum-based chemotherapy are associated with prolonged progression-free survival and overall survival in relapsing $B R C A 1 / B R C A 2$ mutated ovarian cancer patients treated with maintenance olaparib.

6. Garofalo D, Aydin E, Labrador M et al. Real-world data analysis of ovarian cancer (OC) maintenance utilization among maintenance eligible patients. J. Clin. Oncol. 37(Suppl. 15), 5579 (2019).

7. Bartoletti M, Pelizzari G, Gerratana L et al. Bevacizumab or PARP-inhibitors maintenance therapy for platinum-sensitive recurrent ovarian cancer: a network meta-analysis. Int. Mol. Sci. 21(11), 3805 (2020).

- Poly(ADP-ribose) polymerase inhibitors (PARPi) prolonged progression-free survival with respect to bevacizumab in the treatment of platinum-sensitive recurrent ovarian cancer, especially in $B R C A$ mutated patients with no previous exposure to PARPi, but not in BRCA wild-type patients.

8. Moon DH, Lee JM, Noonan AM et al. Deleterious BRCA1/2 mutation is an independent risk factor for carboplatin hypersensitivity reactions. Br. J. Cancer 109, 1072-1078 (2013).

9. Colombo N, Sessa C, du Bois A et al. ESMO-ESGO consensus conference recommendations on ovarian cancer: pathology and molecular biology, early and advanced stages, borderline tumours and recurrent disease. Ann. Oncol. 30, 672-705 (2019).

10. Monk BJ, Herzog TJ, Kaye SB et al. Trabectedin plus pegylated liposomal doxorubicin in recurrent ovarian cancer. J. Clin. Oncol. 28(19), 3107-3114 (2010).

11. Poveda A, Ray-Coquard I, Romero I, Lopez-Guerrero JA, Colombo N. Emerging treatment strategies in recurrent platinum-sensitive ovarian cancer: focus on trabectedin. Cancer Treat. Rev. 40, 366-375 (2014).

12. Krasner CN, Poveda A, Herzog TJ et al. Patient-reported outcomes in relapsed ovarian cancer: results from a randomized Phase III study of trabectedin with pegylated liposomal doxorubicin (PLD) versus PLD alone. Gynecol. Oncol. 127(1), 161-167 (2012).

13. Monk BJ, Ghatage P, Parekh T et al. Effect of BRCA1 and XPG mutations on treatment response to trabectedin and pegylated liposomal doxorubicin in patients with advanced ovarian cancer: exploratory analysis of the Phase 3 OVA-301 study. Ann. Oncol. 26, 914-920 (2015).

14. Pignata S, Scambia G, Mazzei T, Arruti Barbia M, Naglieri E, de Sande LM. Trabectedin plus pegylated liposomal doxorubicin (PLD) in patients with platinum-sensitive recurrent ovarian cancer (PSROC) regardless of prior use of antiangiogenics: first results of an observational, prospective study. Ann. Oncol. 29(Suppl. 8), viii352 (2018).

15. Bouberhan S, Pujade-Lauraine E, Cannistra SA. Advances in the management of platinum-sensitive relapsed ovarian cancer. J. Clin. Oncol. 37, 2424-2436 (2019).

16. Ledermann JA, Gabra H, Jayson GC et al. Inhibition of carboplatin-induced DNA interstrand cross-link repair by gemcitabine in patients receiving these drugs for platinum-resistant ovarian cancer. Clin. Cancer Res. 16, 4899-4905 (2010).

17. Vergote I, Debruyne P, Kridelka F et al. Phase II study of weekly paclitaxel/carboplatin in combination with prophylactic G-CSF in the treatment of gynecologic cancers: a study in 108 patients by the Belgian Gynaecological Oncology Group. Gynecol. Oncol. 138, 278-284 (2015)

18. Coleman RL, Oza AM, Lorusso D et al. Rucaparib maintenance treatment for recurrent ovarian carcinoma after response to platinum therapy (ARIEL3): a randomised, double-blind, placebo-controlled, Phase III trial. Lancet 390, 1949-1961 (2017).

19. Pfisterer J, Shannon CM, Baumann K et al. Bevacizumab and platinum-based combinations for recurrent ovarian cancer: a randomised, open-label, Phase III trial. Lancet Oncol. 21(5), 699-709 (2020).

-. This Phase III trial demonstrated the superiority of carboplatin-pegylated liposomal doxorubicin-bevacizumab to carboplatin-gemcitabine-bevacizumab (standard-of-care) for platinum-sensitive recurrent ovarian cancer. 
20. Rouzier R, Gouy S, Selle F et al. Efficacy and safety of bevacizumab-containing neoadjuvant therapy followed by interval debulking surgery in advanced ovarian cancer: results from the ANTHALYA trial. Eur. J. Cancer 70, 133-142 (2017).

21. Garcia Y, de Juan, Ferré A, Mendiola C et al. Efficacy and safety results from GEICO 1205, a randomized Phase II trial of neoadjuvant chemotherapy with or without bevacizumab for advanced epithelial ovarian cancer. Int. J. Gynecol. Cancer 29, 1050-1056 (2019).

22. Marchetti C. Recurrence of an ovarian cancer patient with limited sensitivity to platinum and without BRCA mutation. Cancer Chemother. Rev. 14, 20-23 (2019).

23. Poveda A, Vergote I, Tjulandin S et al. Trabectedin plus pegylated liposomal doxorubicin in relapsed ovarian cancer: outcomes in the partially platinum-sensitive (platinum-free interval 6-12 months) subpopulation of OVA-301 Phase III randomized trial. Ann. Oncol. 22, 39-48 (2011)

24. Colombo N. Optimizing treatment of the partially platinum-sensitive ovarian cancer patient. Future Oncol. 9(1 Suppl. 12), 19-23 (2013).

25. Colombo N. When nonplatinum is the answer: the role of trabectedin plus pegylated liposomal doxorubicin in recurrent ovarian cancer. Future Oncol. 13(23s), 23-29 (2017).

26. Marth C. Trabectedin + pegylated liposomal doxorubicin in third-line treatment of platinum-sensitive relapsed ovarian cancer: a case study. Expert Rev. Anticancer Ther. 18(Suppl. 1), 19-22 (2018).

27. González A. Increasing the chances for platinum-sensitive ovarian cancer patients. Future Oncol. 9(Suppl. 12), 29-35 (2013).

28. Cecere SC. Multimodal treatment in the therapeutic strategy for an ovarian cancer patient with BRCA mutation. Cancer Chemother. Rev. 14, 60-65 (2019).

29. Murgia V. Management of a platinum-sensitive ovarian cancer patient with BRCA mutation. Cancer Chemother. Rev. 14, 28-32 (2019).

30. Tomao F, D'Incalci M, Biagioli E, Peccatori FA, Colombo N. Restoring platinum sensitivity in recurrent ovarian cancer by extending the platinum-free interval: myth or reality? Cancer 123, 3450-3459 (2017). 